\title{
Transcriptomic analyses reveal molecular mechanisms underlying growth heterosis and weakness of rubber tree seedlings
}

Hong Yang ${ }^{1 \dagger}$, Xuncheng Wang ${ }^{2 \dagger}$, Yongxuan Wei ${ }^{3 \dagger}, Z^{2}$ i Deng ${ }^{1}$, Hui Liu', Jiangshu Chen ${ }^{3}$, Longjun Dai ${ }^{1}$, Zhihui Xia ${ }^{3^{*}}$, Guangming $\mathrm{He}^{2^{*}}$ and Dejun $\mathrm{Li}^{\text {* }^{*}}$

\begin{abstract}
Background: Breeding rubber tree seedling with growth heterosis is vital for natural rubber production. It is the prerequisites for effectively utilizing growth heterosis to elucidate its molecular mechanisms, but the molecular mechanisms remain poorly understood in rubber tree. To elucidate seedling growth heterosis, we conducted comparative transcriptomic analyses between the two hybrids and their parents.

Results: By identifying and comparing differently expressed genes (DEGs), we found that the hybrids (BT 3410 and WC 11) show significantly differential expression profiles from their parents (PR 107 and RRIM 600). In BT 3410-parent triad, 1092 (49.95\%) and 1094 (50.05\%) DEGs indicated clear underdominance or overdominance, respectively. Whereas in WC 11-parent triad, most DEGs (78.2\%, 721) showed low- or high-parent dominance; 160 (17.35\%) exhibited expression patterns that are not statistically distinguishable from additivity, and 8 (0.87\%) and 33 (3.58\%) DEGs exhibited underdominance and overdominance, respectively. Furthermore, some biological processes are differentially regulated between two hybrids. Interestingly, the pathway in response to stimulus is significantly downregulated and metabolic pathways are more highly regulated in BT 3410.

Conclusions: Taken together, the genotypes, transcriptomes and biological pathways (especially, carbohydrate metabolism) are highly divergent between two hybrids, which may be associated with growth heterosis and weakness. Analyzing gene action models in hybrid-parent triads, we propose that overdominance may play important roles on growth heterosis, whereas dominance on hybrid weakness in rubber tree seedlings. These findings bring new insights into our understanding of growth heterosis of rubber tree seedling.
\end{abstract}

Keywords: Hevea brasiliensis, Transcriptome analyses, Growth heterosis, Molecular mechanism, Gene action model

\footnotetext{
*Correspondence: zhxia-111@163.com; heguangming@pku.edu.cn;

dragonldj@163.com; djli.rricatas@gmail.com

${ }^{\dagger}$ Equal contributors

${ }^{3}$ Hainan Key Laboratory for Sustainable Utilization of Tropical Bioresources, College of Agriculture, Hainan University, Haikou, Hainan 570228, China

${ }^{2}$ State Key Laboratory of Protein and Plant Gene Research, Peking-Tsinghua

Center for Life Sciences, School of Advanced Agriculture Sciences and

School of Life Sciences, Peking University, No. 5 Yiheyuan Road, Haidian

District, Beijing 100871, China

${ }^{1}$ Key Laboratory of Biology and Genetic Resources of Rubber Tree, Ministry of

Agriculture, Rubber Research Institute, Chinese Academy of Tropical

Agricultural Sciences, Baodao Xincun, Danzhou, Hainan 571737, China
} 


\section{Background}

Heterosis, or hybrid vigor, refers to the phenomenon that hybrid progeny indicates greater biomass, yield, speed of development, or other agronomic traits than one or both parents [1]. Since heterosis was first described by Charles Darwin [2], it has been widely exploited in plant breeding to dramatically improve the production of crop plants [3]. In contrast to heterosis utilization, the molecular mechanisms involved in this basic biological phenomenon are not well understood.

Three main genetic models are proposed to explain heterosis $[1,4]$. The dominance model states that a complementing action of slightly deleterious recessive alleles contributes to heterosis in $\mathrm{F}_{1}$ hybrid [5]. In contrast to dominance model, overdominance proposes that heterosis comes from favorable allelic interactions at heterozygous loci [6]. Epistasis coming from gene-by-gene interactions is a third genetic model to explain heterosis [7]. In corn and rice, epistasis is associated with inbreeding depression and heterosis [8-10]. Except for some examples from heterozygote advantage and single-locus heterosis, one of three models can not by itself adequately explain heterotic phenomena. Some rice hybrids indicate different genetic models, including epistasis [7], dominance [11], overdominance [12], and overdominance as well as pseudo-dominance [13]. Moreover, similar results have been reported in wheat [14], maize [15-17], upland cotton [18], and Arabidopsis [19, 20]. Differential gene expression in parents and hybrids may be responsible for heterosis [21-28]. In contrast to its parents, a hybrid usually indicates expression levels of genes equal to the mid-parent (additivity, AD), the high or low parent (high or low parent dominance, HPD or LPD), above the high parent (overdominance, OD), or below the low parent (underdominance, UD).

As a critical raw material, natural rubber (NR) can be utilized to manufacture $>40,000$ products. However, it cannot be replaced by synthetic alternatives due to its unique properties including resilience, elasticity, impact and abrasion resistance, efficient heat dispersion and malleability at cold temperature [29]. Among >2000 varieties of rubber-producing plants, the Brazilian rubber tree (Hevea brasiliensis) is the only species cultivated commercially for harvesting NR. The demand for NR has continuously increased due to fast development of the world economy. Breeding high-yield clones is an effective means to meet world demand on NR. Rubber tree breeding has resulted in a gradual increase in NR yields from $650 \mathrm{~kg} / \mathrm{ha}$ in unselected seedlings to $1600 \mathrm{~kg} / \mathrm{ha}$ in optimised hybrid varieties, however the yield of the most productive varieties is still much inferior to the theoretical yield, which was predicted to be $7000-12,000 \mathrm{~kg} / \mathrm{ha}$ [30].
Besides high-yield rubber tree varieties, rubber tree seedling with growth heterosis is vital for NR production. Compared with rubber tree seedlings with growth weakness, those with growth heterosis facilitate themselves to survive various biotic and abiotic stresses; therefore rubber tree seedlings with growth heterosis indicate higher survival ratio than those with growth weakness. Rubber tree usually experiences an initial growth phase varying generally from 5 to 7 years. Trees are tapped when their trunks attain $50 \mathrm{~cm}$ in girth, therefore rubber tree seedlings with growth heterosis can shorten the initial unproductive period. In addition, rubber tree seedling with growth heterosis can shorten the period of seedling cultivation, which can improve the efficiency of breeding seedlings. Some researchers reported that the seedlings of different rubber tree varieties indicated significant difference of growth traits in their immature periods [31-33]. In addition, Sankariammal and Mydin reported that the Wickham $\times$ Amazonian hybrids indicated a significant yield increase (14-82\%) [34]. Although it is vital for NR production to breed rubber tree seedlings with growth heterosis, molecular mechanisms underlying growth heterosis and weakness of rubber tree seedlings are not reported until now.

Here, we generated transcriptome profiles of two rubber tree varieties, and their two $F_{1}$ hybrids which show growth heterosis and weakness, respectively. The gene action models in two hybrid-parent triads were systematically compared to illustrate their relationship with growth heterosis and weakness. We found that underdominance and overdominance expression patterns, and differential expression of genes involved in the pathways of in response to stimulus, and carbohydrate metabolism may contribute to growth heterosis of rubber tree seedlings.

\section{Methods \\ Plant materials, seedlings girth and height, and extraction of RNA}

Five rubber tree varieties, PR 107, RRIM 600, BT 3410, RY 7-33-97, and WC 11 were planted in National Rubber Tree Germplasm Repository, Hainan, China. The leaves from the five varieties were collected with three biological replicates. Two and three replicates were used for RNA-seq and qRT-PCR, respectively. RNA was isolated according to the methods described by Doyle [35]. RNA qualities were assayed with a 2100 Bioanalyzer (Agilent Technologies). In addition, the girth and height of one-year-old seedlings were measured at the height of $1.0 \mathrm{~m}$ and seedling top above the ground level on December 28, 2014, respectively. The seedlings girth and height are shown as mean \pm S.D. Phenotype difference among tree varieties were calculated using $p<0.05$ in 
One-Way ANOVA after Tukey HSD Post-hoc test correction in SPSS (SPSS19.0, IBM, Armonk, New York).

\section{Illumina sequencing, assembly, and annotation}

Sequencing library construction and Illumina sequencing were performed at Novogene Corporation, China. Briefly, mRNA with poly (A) tail was isolated and purified from total RNA with Sera-mag magnetic oligo (dT) beads (Illumina). After the purified mRNA was fragmented into small pieces with $100-400$ bp length, the double-stranded cDNA was synthesized using the SuperScript double-stranded cDNA synthesis kit with random hexamer primers (Illumina). The synthesized cDNA was performed with end-repair and phosphorylation, and then the cDNA fragments were 3' adenylated using Klenow Exo- ( $3^{\prime}$ to $5^{\prime}$ exo minus, Illumina). Next, the ends of the aforementioned 3'-adenylated cDNA fragments were linked with Illumina paired-end adapters. The linked products mentioned above were purified by $2 \%$ agarose gel, and then the cDNA fragments with $\sim 200$ bp were excised from the gel. The purified cDNA templates were enriched by PCR amplification using PCR primer PE 1.0 and 2.0 (Illumina) with phusion DNA polymerase. In the end, the cDNA library with $200 \mathrm{bp}$ insertion fragment was obtained and sequenced using Illumina HiSeqTM 2000. The sequencing workflow was as following: template hybridization, isothermal amplification, linearization, blocking, sequencing primer hybridization, and sequencing on the sequencer for read 1. After finishing the first read, the templates can be regenerated in situ to perform a second read from the opposite end of the fragments. Once the original templates are cleaved and removed, the reverse strands undergo sequencing-by-synthesis. Raw data (raw reads) of fastq format were firstly processed through in-house perl scripts. We filtered out low-quality reads according to three conditions: (1) reads with the adaptor sequence; (2) the percentage of low-quality bases (Qphred $\leq 20$ ) was $>50 \%$ in a read; (3) the percentage of $\mathrm{N}$ bases was $\geq 10 \%$ in a read, and then the paired-end reads were assembled according to the Trinity (V 2.0.6) with min_kmer_cov set to 2 by default and all other parameters set default [36]. The assembled unigenes were functionally annotated based on the following databases: $\mathrm{Nr}$ (NCBI non-redundant protein sequences), Nt (NCBI non-redundant nucleotide sequences), Pfam (Protein family), KOG/COG (Clusters of Orthologous Groups of proteins), Swiss-Prot (A manually annotated and reviewed protein sequence database), KO (KEGG Ortholog database), and GO (Gene Ontology).

\section{qRT-PCR analysis}

The gene-specific primers of 23 unigenes and internal reference gene were listed in Additional file 1: Table S1.
The qRT-PCR conditions were as follows: $94{ }^{\circ} \mathrm{C}$ for $30 \mathrm{~s}$ for denaturation, followed by 45 cycles at $94{ }^{\circ} \mathrm{C}$ for $5 \mathrm{~s}$, $60{ }^{\circ} \mathrm{C}$ for $20 \mathrm{~s}$, and $72{ }^{\circ} \mathrm{C}$ for $20 \mathrm{~s}$. The relative abundance of each unigene was calculated with LightCycler Relative Quantification Software 4.05. All qRT-PCR experiments were repeated 3 times using independent cDNA preparations, and the values are presented as mean \pm S.D. The statistical analysis was performed using SPSS software version 19.0 to find significant difference $(p<0.05)$ with One-Way ANOVA and Tukey HSD Posthoc test correction (IBM, Armonk, New York).

\section{Identification and annotation of DEGs, as well as models of gene action}

The expression level of each gene was calculated using RSEM software (v1.1.17) [37]. With the assembled unigenes as reference, the clean raw reads from four rubber trees varieties were separately realigned to the assembled transcriptome. DESeq R package (1.10.1) was utilized to identify the DEGs with a padj $\leq 0.05$ [38]. If gene expression is different between hybrids and one of their parents, the gene is defined as DEG. The enrichment analysis of GO functional terms and KEGG pathways of the DEGs were calculated as the ratio of the relative occurrence in a set of genes to the relative occurrence in the genome. The statistical significance of the functional enrichment within gene sets was evaluated using the hypergeometric distribution adjusted by the Bonferroni correction for multiple hypotheses testing. Adjusted $P$ values $<0.05$ were considered as significant. In addition, gene expression levels were $\log _{2}$ transformed and used to generate heatmap using R (http://www.r-project.org).

According to the method of Rapp et al. [39], the DEGs among a hybrid-parent triad were classified into 5 possible expression classes of differential expression, that is, expression levels of genes equal to the mid-parent (additivity), the high or low parent (high or low parent dominance), above the high parent (overdominance), or below the low parent (underdominance). In addition, the expression profiles of 23 randomly selected genes were also classified into the aforementioned gene action models in a hybrid-parent triad.

\section{Results}

Transcriptome profiling of two rubber tree varieties and their hybrids

To obtain insight into changes in gene expression and regulatory networks that can affect on growth differences among rubber trees, we generated transcriptome profiles of two rubber tree varieties PR 107, RRIM 600, and their $\mathrm{F}_{1}$ hybrids BT 3410 and WC 11 using Illumina RNA-seq. PR 107 (a primary variety), and RRIM 600 (a secondary variety), are cultivated from the unselected seedling of clone LCB 510 and the offsprings of two 
primary clones (Tjir 1 and PB 86), respectively. BT 3410, RY 7-33-97 and WC 11 are generated by selecting from the $F_{1}$ offsprings of PR 107 and RRIM 600 (Fig. 1a). The seedling girth of RRIM 600 is significantly thicker than that of PR 107 (Fig. 1b and Additional file 2: Table S2). The seedling height of RRIM 600 is taller than that of PR 107, but the difference is not statistically significant (Fig. 1c and Additional file 2: Table S2). Compared to their parents, BT 3410 is significantly thicker and taller than RRIM 600, whereas WC 11 is shorter and thinner than PR 107 (Fig. 1b, c and Additional file 2: Table S2), indicating growth heterosis and weakness in BT 3410 and WC 11, respectively. Different from BT 3410 and WC 11, the seedling girth and height of RY 7-33-97 are between PR 107 and RRIM 600 (Fig. 1b and c, Supplementary Dataset 2).

Leaves from one-year-old seedling were used to generate RNA-seq libraries. After the adaptors, low-quality and contaminated reads were removed, a total of $331,788,346$ RNA-seq reads were obtained from PR 107, RRIM 600, BT 3410, and WC 11. We obtained 152,231 unigenes by using high-quality reads from all four rubber tree varieties for de novo transcriptome assembling. The average and N50 lengths of the assembled unigenes are $731 \mathrm{bp}$ and $1193 \mathrm{bp}$, respectively. Of 152,231 unigenes, 13,277 ( 8.72\%) and 96,971 ( 63.69\%) indicate homology to the genes or their protein products from all or at least one of seven databases including NCBI Nr and Nt, Pfam, KOG/COG, Swiss-prot, KEGG, and GO with e-value cutoff of $1 \mathrm{e}-5$, respectively (Table 1 ). With the 152,231 assembled unigenes as reference, the highquality clean reads from the four varieties were separately realigned, and gene expression was calculated based on mapped reads in each variety. We found that there are 58,210,60,276, 66,514, and 62,723 genes that were expressed in PR 107, RRIM 600, BT 3410, and WC 11 , respectively, and 43,547 genes were expressed in all four rubber tree varieties (Fig. 2).

\section{BT 3410 and WC 11 show significantly different models of gene action}

Next, we investigated the variation in gene expression among hybrids and parents. With a padj $<0.05$ as a threshold, approximately 1.95\% (2975) unigenes were identified as the differently expressed genes (DEGs) between RRIM 600 and PR 107, and about 6.17\% (9396) and $6.37 \%$ (9696) unigenes were identified as the DEGs among a BT 3410- or WC 11-parent triad, respectively. We investigated the expression profiles of DEGs in two hybrid-parent triads using hierarchical clustering. As shown in Fig. 3a, gene expression profiles between PR 107 and RRIM 600 differ from each other. BT 3410 and
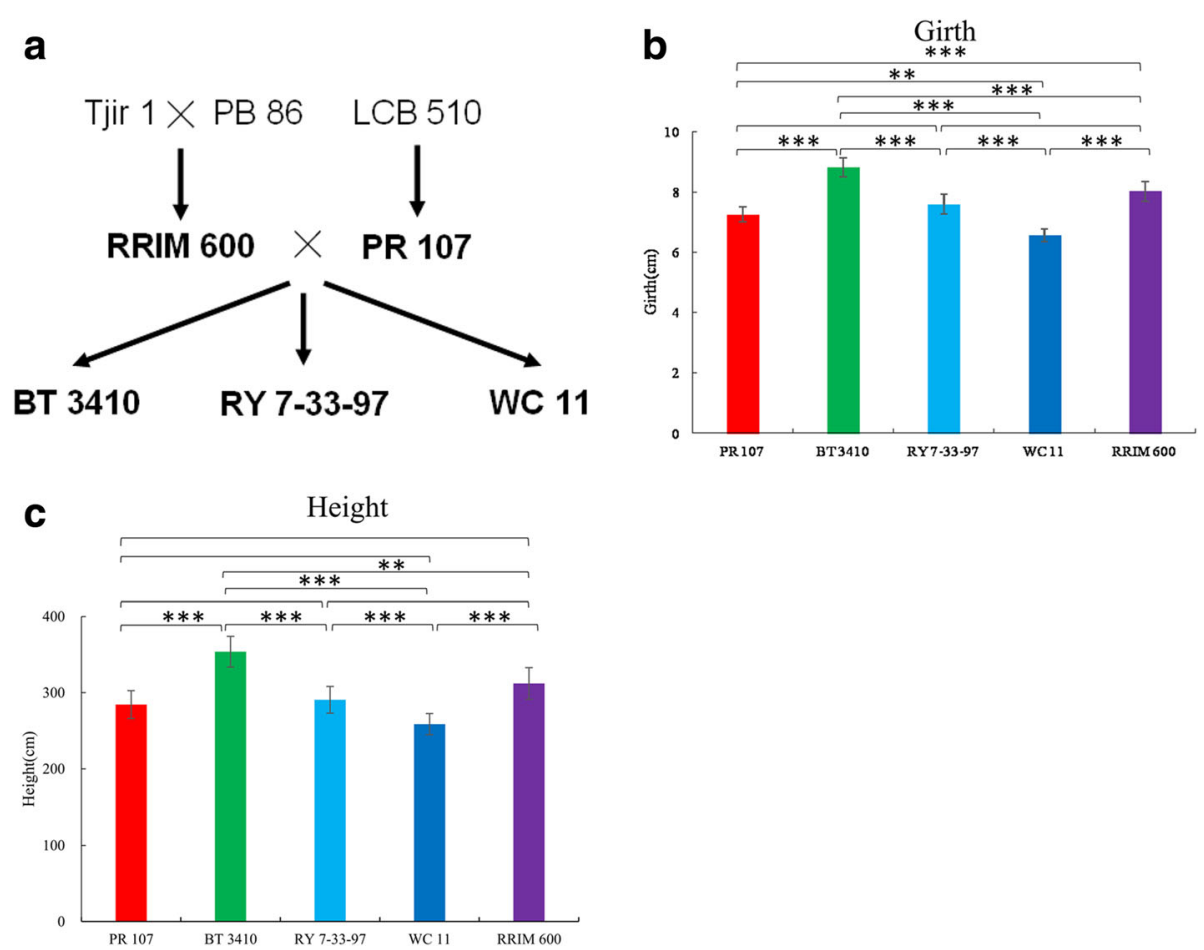

Fig. 1 Genetic background, seedling girth and height of five rubber tree varieties. a Genetic background of rubber tree varieties analyzed in this study. Female parent of each cross is listed first. The five varieties analyzed in this study are in bold type. b, c Seedling girth and height of three hybrids and their parents. The data is firstly analyzed with One-Way ANOVA, and then analyzed with Tukey HSD Post-hoc test. Three and two asterisks represent $p<0.0001$ and $p<0.001$, respectively 
Table 1 Annotated percentage of unigenes in different databases

\begin{tabular}{lll}
\hline Items & Number of Unigenes & Percentage (\%) \\
\hline Annotated in NR & 82,098 & 53.92 \\
Annotated in NT & 75,970 & 49.9 \\
Annotated in KO & 28,265 & 18.56 \\
Annotated in SwissProt & 57,008 & 37.44 \\
Annotated in PFAM & 49,901 & 32.77 \\
Annotated in GO & 52,096 & 34.22 \\
Annotated in KOG & 28,773 & 18.9 \\
Annotated in all Databases & 13,277 & 8.72 \\
Annotated in at least one & 96,971 & 63.69 \\
Database & & 100 \\
Total Unigenes & 152,231 & \\
\hline
\end{tabular}

WC 11 show significantly differential expression profile from both parents, which, to some extent, may explain growth heterosis and weakness in BT 3410 and WC 11, respectively.

We further investigated the models of gene action in two hybrid-parent triad. In BT 3410, 2186 genes exhibit nonadditive model and no gene exhibits additive model. Of 2186 genes, 1092 (49.95\%) and 1094 (50.05\%) show UD and OD patterns, respectively (Fig. $3 \mathrm{~b}$ and Additional file 3: Table S3). In WC 11, 992 genes could be classified into five gene model, among which only 8 $(0.87 \%)$ and $33(3.58 \%)$ exhibited UD and OD patterns, respectively. A large proportion of the genes (78.2\%, 721) showed dominance models. Of these 721 genes, 269 and 452 exhibited HPD and LPD patterns, respectively. In addition, 160 genes (17.35\%) exhibited expression patterns that are not statistically distinguishable from $\mathrm{AD}$ (Fig. 3b and Additional file 4: Table S4). We randomly selected 23 genes to validate their gene action

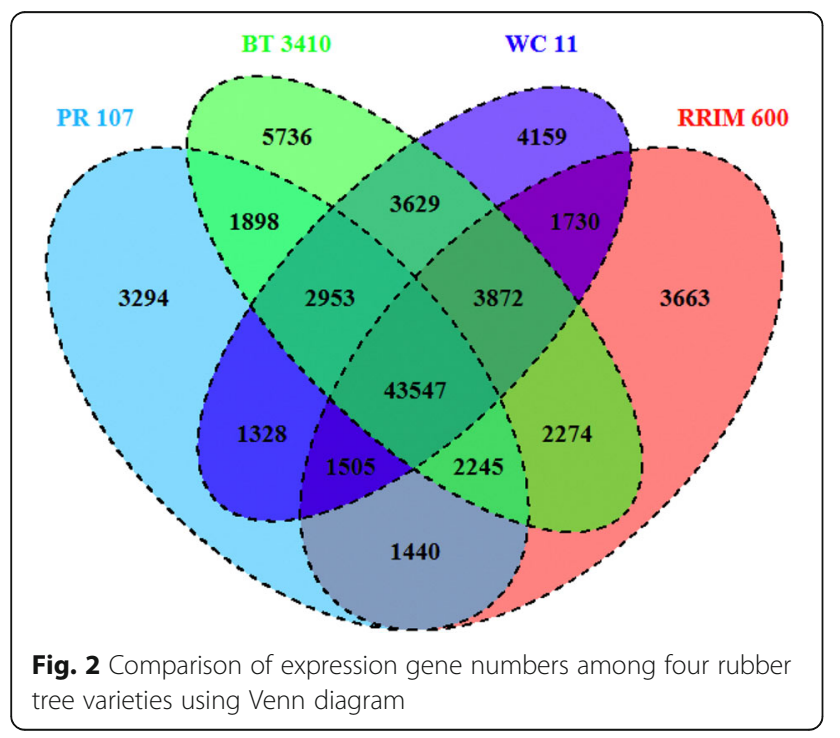

models among two hybrid-parent triads using qRT-PCR. Among 23 genes (32 gene action models) deduced from RNA-seq data, all were confirmed by qRT-PCR (Fig. 4), suggesting that the gene action models analyzed in two hybrid-parent triads were highly reliable in our study. In addition, gene action models of the 23 genes were also analyzed in RY 7-33-97 and its parents by qRT-PCR. Among 20 genes indicating gene action models, 7, 4, 2, 1 , and 6 genes indicated HPD, LPD, OD, AD, and UD gene action models in RY 7-33-97 and its parent triad, respectively (Fig. 4c). These results indicated that BT 3410, RY 7-33-97 and WC 11 show significantly different gene action models although they are the offsprings of the same parents, which may contribute to growth heterosis and weakness of seedlings in these rubber tree hybrids, respectively.

\section{Biological processes are differentially regulated between BT 3410 and WC 11}

To explore the biological implication of changed gene expression in the two hybrids, we further investigated the Gene Ontology (GO) functional categories of genes with different expression patterns. In BT 3410, four GO terms including single-organism process, metabolic process, localization, and cellular process are significantly enriched for genes both with UD and OD patterns; GO term response to stimulus is significantly enriched specifically for genes with UD pattern, whereas seven other GO terms (biological regulation, cellular component organization or biogenesis, regulation of biological process, locomotion, positive regulation of biological process, biological adhesion, and multi-organism process) are enriched specifically for genes with OD pattern (Table 2). In WC 11, two GO terms (multi-organism process, and reproductive process) are significantly enriched for genes both with $\mathrm{AD}$ and LPD; two GO terms (multicellular organismal process, and response to stimulus), two GO terms (single-organism process, and immune system process), and two other GO terms (metabolic process, and positive regulation of biological process) are significantly enriched specifically for genes with AD, UD, and LPD patterns, respectively (Table 2). In addition, no GO term is significantly enriched for genes with HPD. Table 2 also shows that there are 5 GO terms (single-organism process, metabolic process, response to stimulus, multi-organism process and positive regulation of biological process) shared by the two hybrids. Interestingly, the genes involved in response to stimulus are significantly down-regulated (with UD pattern) in BT 3410, which potentially contribute to the seedling heterosis in this hybrid through tradeoff between stress response and growth. By contrast, in WC 11 exhibiting hybrid weakness, the genes involved in response to stimulus are equal to mid-parent values (with 

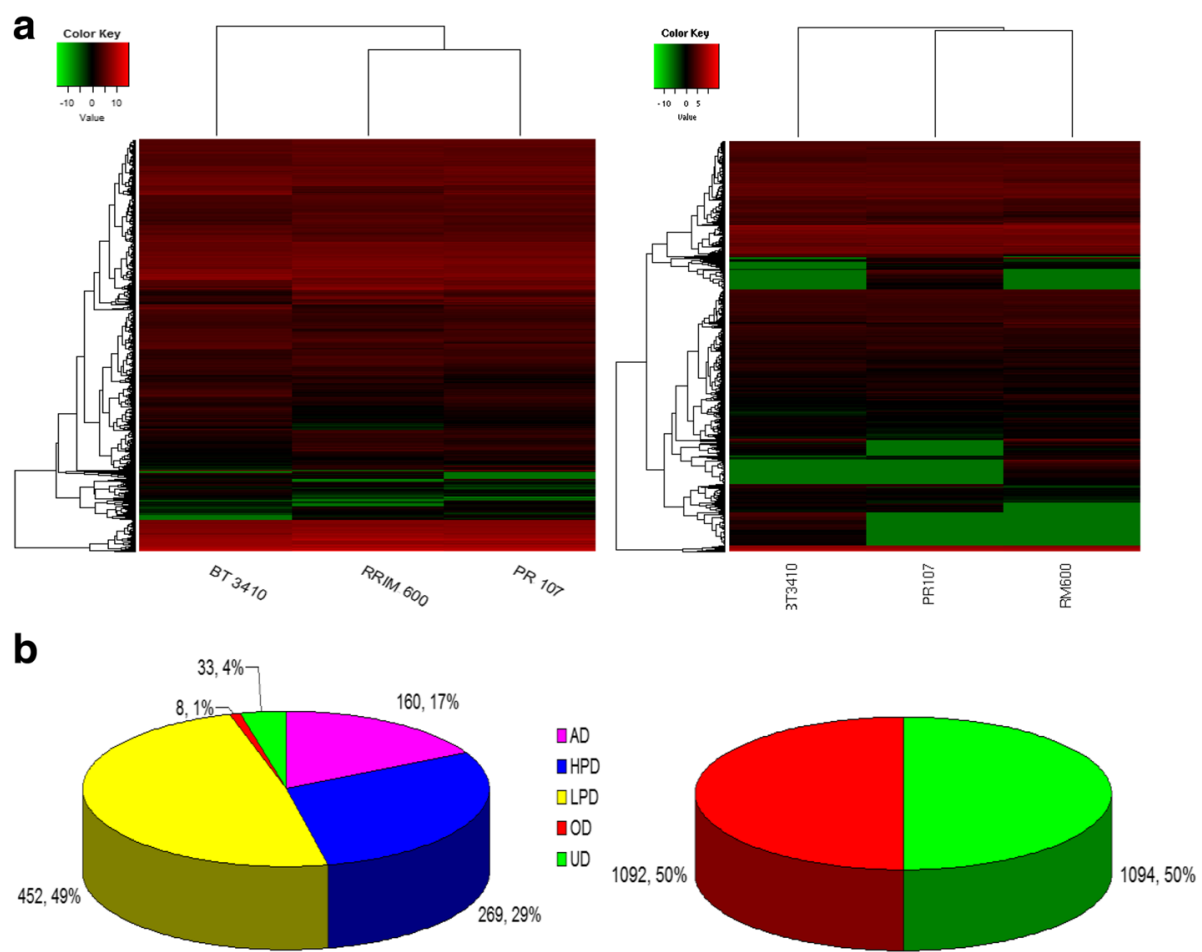

Fig. 3 Heat map and gene action models in two hybrid-parent triads. a Heat map generated from gene expression in two hybrid-parent triads. $\mathbf{b}$ Sector graph of gene action models in WC 11 (left) and BT 3410 (right)

AD pattern). Furthermore, genes involved in positive regulation of biological process are significantly upregulated (with OD pattern) in BT 3410, whereas are significantly down-regulated (with LPD pattern) in WC 11. Genes involved in metabolic process are up- or down-regulated in BT 3410, whereas in WC 11 only down-regulation is observed for genes in this process. Difference in the regulation of these pathways may also contribute to the growth difference between two rubber tree hybrids.

\section{Metabolic pathways are highly regulated in BT $\mathbf{3 4 1 0}$}

We also classified DEGs with different models of gene action in hybrids according to their KEGG pathways. As shown in Table 3, the DEGs in BT 3410 and in WC 11 are assigned to 31 and 27 KEGG pathways, respectively. With qualue $<0.05$ as a threshold, 8 and 2 KEGG pathways are significantly enriched in BT 3410 and WC 11, respectively. Interestingly, all 10 enriched KEGG pathways in two hybrids belong to metabolism. Two KEGG pathways, amino acid metabolism and biosynthesis of other secondary metabolites, are enriched both in BT 3410 and WC 11. Six KEGG pathways including carbohydrate metabolism, lipid metabolism, metabolism of cofactors and vitamins, xenobiotics biodegradation and metabolism, metabolism of other amino acids, and overview are enriched specifically in BT 3410 . It should be noted that carbohydrate metabolism is enriched in BT 3410 , but not in WC 11. Carbohydrate metabolism is very important for generating cellular energy and various metabolites that provide material and energy to support plant growth and development. Therefore, the DEGs in carbohydrate metabolism pathway may directly contribute to the growth heterosis in rubber tree.

\section{Discussion}

RNA sequencing (RNA-Seq) is a valuable tool for profiling expressed genes in plants and other organisms [4042]. Leaf is an important tissue where photosynthesis and transpiration occur in rubber tree. The product of photosynthesis can provide material basis for rubber tree growth and development. Compared with rubber tree latexes [43-50] and barks [51-54], the research on transcriptome sequencing and assembly of rubber tree leaves was very limited. A total of 98,796 unigenes were obtained via the de novo assembly of RNA-seq data from rubber tree leaves [55]. The number of unigenes generated in this study was more than that reported by Fang et al. [55]. The N50 length of the unigenes in the present study was $1139 \mathrm{bp}$, which was longer than that obtained by Fang et al. [55]. Our leaf transcriptome data was submitted to NCBI database, which will further enrich the rubber tree transcriptome data. In addition, it will be 


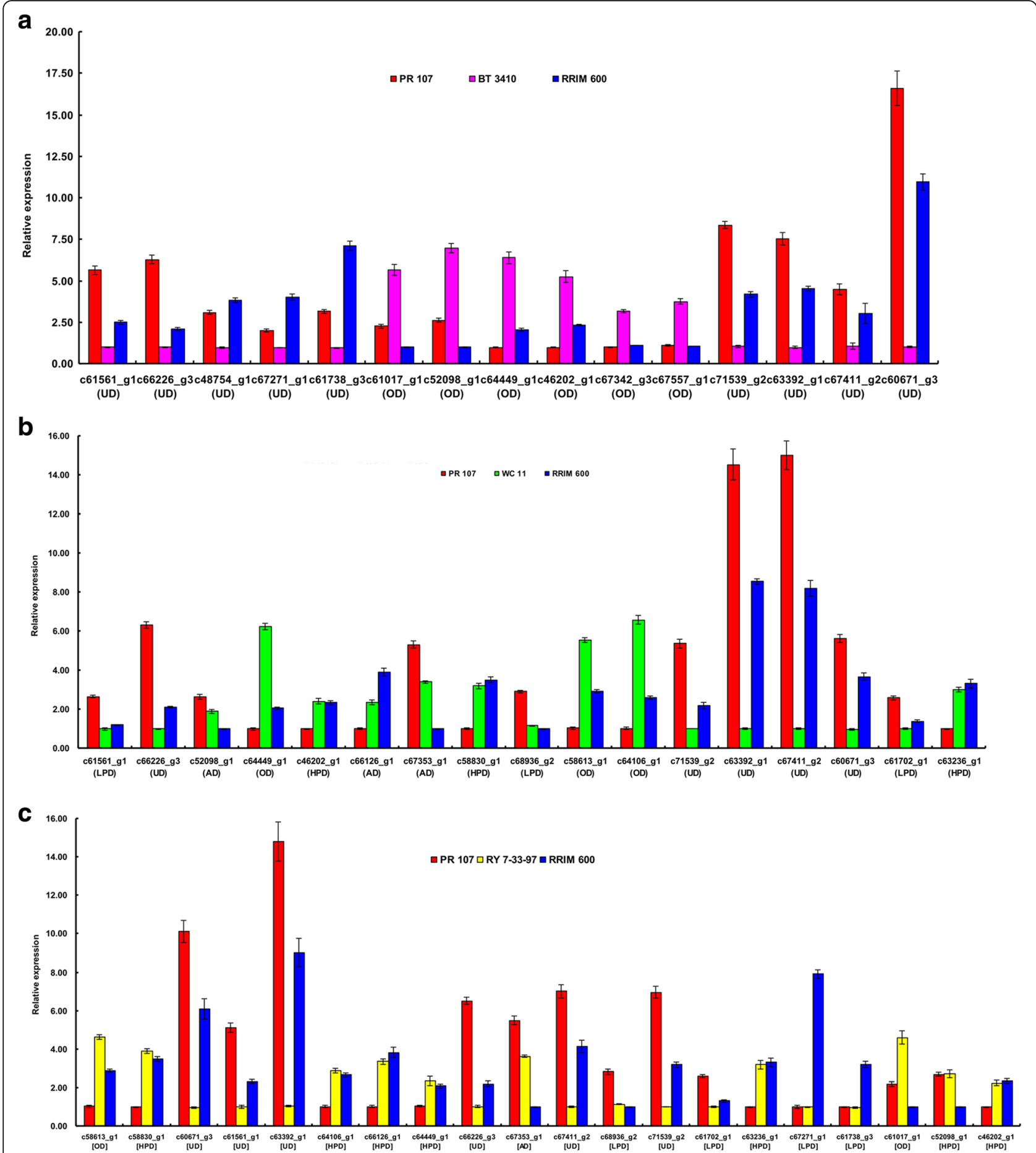

Fig. 4 Gene expression profiles of 23 genes generated from qRT-PCR in three hybrid-parent triads. $\mathbf{a}$, $\mathbf{b}$, and $\mathbf{c}$ present gene expression profiles in BT 3410-, WC 11-, and RY 7-33-97-parent triads, respectively. AD, OD, UD, LPD, and HPD in parentheses and bracket refer to gene action models generated from RNA-seq and qRT-PCR analyses, respectively. One-Way ANOVA followed with Tukey HSD Post-hoc test and $p<0.05$ were used to calculating gene expression difference

helpful for developing molecular markers, improving transcriptome and genome assembly, gene cloning, etc.

Although three $F_{1}$ progenies are generated form the same parents (RRIM 600 and PR 107), the $\mathrm{F}_{1}$ progenies,
BT 3410, WC 11, and RY 7-33-97, show growth heterosis, growth weakness, and in between (represented by girth and height) relative to their parents, respectively. The growth differences among three $F_{1}$ progenies may 
Table 2 Enriched GO terms within biological process in BT 3410 and WC 11

\begin{tabular}{|c|c|c|c|c|c|c|c|}
\hline GO accession & Terms & Genes No. in this term & Genes No. in all terms & pvalue & qvalue & Models & Varieties \\
\hline GO:0044699 & single-organism process & 463 & 825 & $1.11 \mathrm{E}-16$ & $3.16 \mathrm{E}-15$ & UD & BT 3410 \\
\hline GO:0008152 & metabolic process & 555 & 825 & $2.55 \mathrm{E}-10$ & 4.85E-09 & & \\
\hline GO:0050896 & response to stimulus & 130 & 825 & 0.00027 & 0.00193 & & \\
\hline GO:0051179 & localization & 156 & 825 & 0.00173 & 0.00987 & & \\
\hline GO:0009987 & cellular process & 494 & 825 & 0.00736 & 0.03812 & & \\
\hline GO:0044699 & single-organism process & 418 & 824 & $2.02 \mathrm{E}-07$ & 5.75E-06 & OD & \\
\hline GO:0008152 & metabolic process & 535 & 824 & 7.76E-07 & 1.47E-05 & & \\
\hline GO:0009987 & cellular process & 515 & 824 & $3.41 \mathrm{E}-05$ & 0.00024 & & \\
\hline GO:0065007 & biological regulation & 187 & 824 & 3.33E-05 & 0.00024 & & \\
\hline GO:0071840 & $\begin{array}{l}\text { cellular component } \\
\text { organization or biogenesis }\end{array}$ & 102 & 824 & 0.00017 & 0.00095 & & \\
\hline GO:0051179 & localization & 157 & 824 & 0.00122 & 0.00366 & & \\
\hline GO:0050789 & regulation of biological process & 164 & 824 & 0.00158 & 0.00451 & & \\
\hline GO:0040011 & locomotion & 13 & 824 & 0.00402 & 0.01091 & & \\
\hline GO:0048518 & $\begin{array}{l}\text { positive regulation of } \\
\text { biological process }\end{array}$ & 16 & 824 & 0.00588 & 0.01525 & & \\
\hline GO:0022610 & biological adhesion & 11 & 824 & 0.0113 & 0.028 & & \\
\hline GO:0051704 & multi-organism process & 47 & 824 & 0.01814 & 0.03998 & & \\
\hline GO:0051704 & multi-organism process & 16 & 106 & $2.41 \mathrm{E}-06$ & 0.00014 & $A D$ & WC 11 \\
\hline GO:0032501 & multicellular organismal process & 8 & 106 & 7.85E-05 & 0.00224 & & \\
\hline GO:0022414 & reproductive process & 4 & 106 & 0.00049 & 0.00929 & & \\
\hline GO:0050896 & response to stimulus & 21 & 106 & 0.00579 & 0.04127 & & \\
\hline GO:0051704 & multi-organism process & 27 & 325 & 0.00036 & 0.00508 & LPD & \\
\hline GO:0022414 & reproductive process & 7 & 325 & 0.00091 & 0.00861 & & \\
\hline GO:0008152 & metabolic process & 212 & 325 & 0.00082 & 0.00861 & & \\
\hline GO:0048518 & $\begin{array}{l}\text { positive regulation of } \\
\text { biological process }\end{array}$ & 8 & 325 & 0.00706 & 0.03657 & & \\
\hline GO:0044699 & single-organism process & 16 & 23 & 0.00201 & 0.03818 & UD & \\
\hline GO:0002376 & immune system process & 1 & 23 & 0.00648 & 0.04106 & & \\
\hline
\end{tabular}

attribute to their parents' heterozygosity. We found that a large proportion of genes (>82\%) indicate nonadditive models in the two hybrids from heterozygous crossing, which is consistent with Hedgecock et al. [56], Wei et al. [23], Song et al. [57], and Li et al. [25], but differ from Swanson-Wagner et al. [15] and Stupar and Springer [58] who found very little gene expressions that were nonadditive or that exceeded parental levels in hybrids from homozygous crossing. On the other hand, the two hybrids also show different models of gene action. All the genes exhibit OD or UD actions in BT 3410 generated from heterozygous crossing, suggesting that OD or UD may play critical important roles in growth heterosis. As for the hybrids generated from homozygous crossing, Hedgecock et al. [56] proposed that the hybrid family with the greater degree of growth heterosis showed a greater OD proportion. Li et al. also reported that higher proportions of nonadditive and expression higher or lower than either parent were found in heterotic hybrids compared to a non-heterotic hybrid [59]. Different from BT 3410, additive and nonadditive models are separately $\sim 17.35 \%$ and $82.65 \%$ in WC 11 . Of the genes exhibiting nonadditive, $\sim 78.2 \%$ exhibit LPD or HPD. Moreover, UD and OD are also observed in WC 11. Our results are broadly similar to those of Song et al. [57] and Li et al. [25], but their results are form the hybrids of homozygous crossing and the hybrids indicate heterosis. However, the hybrid WC 11 shows growth weakness, suggesting that a large proportion of dominance models may be associated with growth weakness of rubber tree seedlings. Additionally, the gene action models of the 23 genes randomly selected in RY 7-3397 were different from those of WC 11 and BT 3401. Although the 23 gene action models could not necessarily represent the gene action models of RY 7-33-97 on transcriptome level, the seedling girth and height of RY 
Table 3 The KEGG functional categories of the DEGs between two hybrid-parent triads

\begin{tabular}{|c|c|c|}
\hline \multirow[t]{2}{*}{ Functional categories } & \multicolumn{2}{|c|}{ The gene number } \\
\hline & BT 3410 & WC 11 \\
\hline \multicolumn{3}{|l|}{ Metabolism } \\
\hline $\begin{array}{l}\text { Biosynthesis of other secondary } \\
\text { metabolites }\end{array}$ & $42^{\mathrm{a}}$ & $14^{\mathrm{a}}$ \\
\hline Amino acid metabolism & $69^{a}$ & $20^{\mathrm{a}}$ \\
\hline Carbohydrate metabolism & $90^{\mathrm{a}}$ & 13 \\
\hline Overview & $67^{\mathrm{a}}$ & 6 \\
\hline $\begin{array}{l}\text { Metabolism of cofactors and } \\
\text { vitamins }\end{array}$ & $33^{\mathrm{a}}$ & 4 \\
\hline Lipid metabolism & $44^{\mathrm{a}}$ & 13 \\
\hline Metabolism of other amino acids & $29^{a}$ & 6 \\
\hline $\begin{array}{l}\text { Xenobiotics biodegradation and } \\
\text { metabolism }\end{array}$ & $13^{\mathrm{a}}$ & 5 \\
\hline $\begin{array}{l}\text { Metabolism of terpenoids and } \\
\text { polyketides }\end{array}$ & 18 & 7 \\
\hline Energy metabolism & 47 & 9 \\
\hline Nucleotide metabolism & 15 & 3 \\
\hline $\begin{array}{l}\text { Glycan biosynthesis and } \\
\text { metabolism }\end{array}$ & 3 & 1 \\
\hline \multicolumn{3}{|l|}{ Cellular Processes } \\
\hline Cell growth and death & 27 & 6 \\
\hline Transport and catabolism & 32 & 2 \\
\hline Cell motility & 5 & 0 \\
\hline Cellular commiunity & 9 & 1 \\
\hline \multicolumn{3}{|l|}{ Environmental Information Processing } \\
\hline Membrane transport & 3 & 1 \\
\hline Signal transduction & 39 & 15 \\
\hline \multicolumn{3}{|l|}{ Genetic Information Processing } \\
\hline Replication and repair & 11 & 5 \\
\hline Folding, sorting and degradation & 23 & 9 \\
\hline Transcription & 2 & 4 \\
\hline Translation & 17 & 2 \\
\hline \multicolumn{3}{|l|}{ Organismal Systems } \\
\hline Digestive system & 10 & 2 \\
\hline Endocrine system & 25 & 4 \\
\hline Development & 4 & 0 \\
\hline Sensory system & 1 & 0 \\
\hline Circulatory system & 2 & 2 \\
\hline Environmental adaptation & 11 & 6 \\
\hline Excretory system & 2 & 0 \\
\hline Immune system & 10 & 5 \\
\hline Nervous system & 12 & 5 \\
\hline
\end{tabular}

adenote function categories of the DEGs significantly enriched with $q$ value $<0.05$

7-33-97 are indeed between PR 107 and RRIM 600. These results suggest that heterosis from homozygous and heterozygous crossings may possess some similarities and differences in models of gene action, which need further be validated in future experiments.

Different combinations among altered activity of biological processes can be charge of heterotic phenotypes [3, 60], which was further validated by Groszmanna et al. in Arabidopsis $\mathrm{F}_{1}$ hybrids. They suggested that the altered expression patterns of defense and stress response genes contributed to the greater growth of the hybrids [61]. In intraspecific hybrids, genome-wide expression of biotic and abiotic stressresponsive genes is diurnally repressed, which correlates with biomass heterosis and biomass quantitative trait loci [62]. It is worth noting that the DEGs significantly enriched in response to stimulus are separately classified in UD and AD in BT 3410 and WC 11. Given the antagonistic relationship between plant growth and defense responses [63-65], we suggest that these downregulated expressions of genes involved in response to stimulus may contribute to seedlings growth heterosis of the hybrids. Consistent with the aforementioned speculation, the genes involved in response to stimulus indicate $\mathrm{AD}$ model in WC 11 showing seedlings growth weakness. Besides response to stimulus terms, Wang et al. also found that the DEGs involved in metabolic process contribute to growth heterosis in both the hybrid and the hybrid mimic lines [66]. In accordance with Wang et al. [66], we found that the DEGs were also significantly enriched in metabolic process in BT 3410 (UD or OD) and WC 11 (LPD).

Lisec et al. reported that primary metabolism is a network that is closely linked to plant growth and development [67]. In a parallel study, they found that a linear combination of metabolite levels was shown to significantly correlate with biomass heterosis [68]. Consistent with the aforementioned results, we found that the DEGs in two hybrids significantly enriched in metabolism that is exclusive among five main KEGG pathways. We found that $87.5 \%$ and $100 \%$ enriched-KEGG pathways are primary or secondary metabolism in hybrids BT 3410 and WC 11, respectively. Yield heterosis-associated genes significantly enriched in carbohydrate metabolism in maize [17]. In addition, carbohydrate metabolism was identified as significant difference between LYP9 and its parents $[23,57,69,70]$. In our study, carbohydrate metabolism significantly enriched in BT 3410 (growth heterosis), but not in WC 11 (growth weakness), suggesting that carbohydrate metabolism may contribute to growth heterosis. Given the aforementioned results, we suggest that the DEGs involved in the enriched carbohydrate metabolism may work together to be responsible for seedlings growth heterosis of rubber tree. The aforementioned results indicate that the hybrids generated from homozygous and heterozygous crossings may show the heterosis by 
regulating similar GO terms and KEGG pathways. Of course, this speculation need be further testified.

Analyzing gene action models between the two hybrids and their parents, we propose that overdominance (OD or UD) may play important roles on growth heterosis, whereas dominance on hybrid weakness in rubber tree seedlings. It is the first time to analyze growth heterosis and weakness on transcriptome level in rubber tree seedlings. These findings bring new insights into understanding growth heterosis of rubber tree seedling.

\section{Conclusions}

We found that the genotypes, transcriptomes and biological pathways (especially, carbohydrate metabolism) were highly divergent between two hybrids, which may be associated with growth heterosis and weakness. Analyzing gene action models between the two hybrids and their parents, we propose that overdominance may play important roles on growth heterosis, whereas dominance on hybrid weakness in rubber tree seedlings.

\section{Additional files}

Additional file 1: Table S1. Primer pairs designed for validating gene expression. (XLSX $25 \mathrm{~kb}$ )

Additional file 2: Table S2. Seedling girth and height of five rubber tree varieties. (XLSX 8 kb)

Additional file 3: Table S3. Gene action models in BT 3410-parent triad. (XLSX $478 \mathrm{~kb}$ )

Additional file 4: Table S4. Gene action models in WC 11-parent triad. (XLSX $205 \mathrm{~kb}$ )

\section{Abbreviations}

AD: Additivity; GO: Gene ontology; HPD: High parent dominance; KO: KEGG ortholog database; KOG/COG: Clusters of orthologous groups of proteins; LPD: Low parent dominance; NR: Natural rubber; Nr: NCBI non-redundant protein sequences; Nt: NCBI non-redundant nucleotide sequences; OD: Overdominance; Pfam: Protein family; UD: Underdominance

\section{Acknowledgements}

The authors would like to thank National Rubber Tree Germplasm Repository, Hainan, China to provide experimental materials. We also thank Novogene Corporation for assistance with the raw data processing and bioinformatics analyses.

\section{Funding}

This research was supported by the earmarked funds from National Natural Science Foundation of China (31570684 and 31270651) and Fundamental Research Funds for Rubber Research Institute, CATAS (1630022015003 and 1630022014006)

\section{Availability of data and materials}

Sequence data from this article were submitted to the EMBL/GenBank data libraries under accession number GEIA01000000.

\section{Authors' contributions}

LDJ, HGM and XZH designed the research. LDJ, WXC, WYX, YH, DZ, LH and CJS performed research and analyzed data. LDJ and HGM wrote and the article. LDJ, XZH, WXC and HGM revised the article. All authors read and approved the final manuscript.
Ethics approval and consent to participate

Not applicable.

\section{Consent for publication}

Not applicable.

Competing interests

The authors declare that they have no competing interests.

\section{Publisher's Note}

Springer Nature remains neutral with regard to jurisdictional claims in published maps and institutional affiliations.

Received: 3 September 2017 Accepted: 4 December 2017

Published online: 09 January 2018

References

1. Birchler JA, Auger DL, Riddle NC. In search of the molecular basis of heterosis. Plant Cell. 2003:15:2236-9.

2. Darwin CR. The effects of cross- and self-fertilization in the vegetable kingdom. New York: D. Appleton; 1876.

3. Schnable PS, Springer NM. Progress toward understanding heterosis in crop plants. Annu Rev Plant Biol. 2013:64:71-88.

4. Birchler JA, Yao H, Chudalayandi S. Unraveling the genetic basis of hybrid vigor. Proc Natl Acad Sci U S A. 2006:103:12957-8.

5. Jones DF. Dominance of linked factors as a means of accounting for heterosis. Genetics. 1917:2:466-79.

6. Crow JF. Alternative hypothesis of hybrid vigor. Genetics. 1948;33:477-87.

7. Yu SB, Li JX, Xu CG, Tan YF, Gao YJ, Li XH, et al. Importance of epistasis as the genetic basis of heterosis in an elite rice hybrid. Proc Natl Acad Sci U S A. 1997;94:9226-31.

8. Stuber CW, Lincoln SE, Wolff DW, Helentjaris T, Lander ES. Identification of genetic factors contributing to heterosis in a hybrid from two elite maize inbred lines using molecular markers. Genetics. 1992;132:823-39.

9. Li ZK, Luo L, Mei HW, Wang DL, Shu QY, Tabien R, et al. Overdominant epistatic loci are the primary genetic basis of inbreeding depression and heterosis in rice. I. Biomass and grain yield. Genetics. 2001;158:1737-53.

10. Luo LJ, Li ZK, Mei HW, Shu OY, Tabien R, Zhong DB, et al. Overdominant epistatic loci are the primary genetic basis of inbreeding depression and heterosis in rice. II. Grain yield components. Genetics. 2001;158:1755-71.

11. Li LZ, Lu KY, Chen ZM, Mu TM, Hu ZL, Li XQ. Dominance, overdominance and epistasis condition the heterosis in two heterotic rice hybrids. Genetics. 2008;180:1725-42

12. Xiao JH, Li JM, Yuan LP, Tanksley SD. Dominance is the major genetic basis of heterosis in rice as revealed by QTL analysis using molecular markers. Genetics. 1995;140:745-54.

13. Zhou G, Chen Y, Yao W, Zhang CJ, Xie WB. HuaJP, et al. genetic composition of yield heterosis in an elite rice hybrid. Proc Natl Acad Sci U S A. 2012;109:15847-52.

14. Wang Z, Ni Z, Wu H, Nie X, Sun Q. Heterosis in root development and differential gene expression between hybrids and their parental inbreds in wheat (Triticum aestivum L.). Theor Appl Genet. 2006;113(7):1283-94.

15. Swanson-Wagner RA, Jia Y, DeCook R, Borsuk LA, Nettleton D, Schnable PS, et al. All possible modes of gene action are observed in a global comparison of gene expression in a maize F1 hybrid and its inbred parents. Proc Natl Acad Sci U S A. 2006;103:6805-10.

16. Stupar RM, Hermanson PJ, Springer NM. Nonadditive expression and parent-of-origin effects identified by microarray and allele-specific expression profiling of maize endosperm. Plant Physiol. 2007;145:411-25.

17. Zhang TF, Li B, Zhang DF, Jia GQ, Li ZY, Wang SC. Genome-wide transcriptional analysis of yield and heterosis-associated genes in maize (Zea mays L.). J Integr Agri. 2012;11:1245-56.

18. Liang QZ, Shang LG, Wang YM, Hua J. Partial dominance, overdominance and epistasis as the genetic basis of heterosis in upland cotton (Gossypium hirsutum L.). PLoS One. 2015:10:e0143548.

19. Syed $\mathrm{NH}$, Chen ZI. Molecular marker genotypes, heterozygosity and genetic interactions explain heterosis in Arabidopsis thaliana. Heredity. 2005:94:295-304.

20. Vuylsteke M, van Eeuwijk F, Van Hummelen P, Kuiper M, Zabeau M. Genetic analysis of variation in gene expression in Arabidopsis thaliana. Genetics. 2005;171:1267-75. 
21. Song RT, Messing J. Gene expression of a gene family in maize based on noncollinear haplotypes. Proc Natl Acad Sci U S A. 2003;100:9055-60.

22. Semel $Y$, Nissenbaum J, Menda N, Zinder M, Krieger U, Issman N, et al. Overdominant quantitative trait loci for yield and fitness in tomato. Proc Natl Acad Sci U S A. 2006;103:12981-6.

23. Wei G, Tao Y, Liu GZ, Chen C, Luo RY, Xia HA, et al. A transcriptomic analysis of superhybrid rice LYP9 and its parents. Proc Natl Acad Sci U S A. 2009;106:7695-701.

24. Li AL, Liu DC, Wu J, Zhao XB, Hao M, Geng SF, et al. mRNA and small RNA transcriptomes reveal insights into dynamic homoeolog regulation of allopolyploid heterosis in nascent hexaploid wheat. Plant Cell. 2014;26:1878-900.

25. Li DJ, Zeng RZ, Li Y, Zhao MM, Chao JQ, Li Y, et al. Gene expression analysis and SNP/InDel discovery to investigate yield heterosis of two rubber tree $F_{1}$ hybrids. Sci Rep. 2016;6:24984.

26. Li DY, Huang ZY, Song SH, Xin YY, Mao DH, Lv QM, et al. Integrated analysis of phenome, genome, and transcriptome of hybrid rice uncovered multiple heterosisrelated loci for yield increase. Proc Natl Acad Sci U S A. 2016;113:E6026-35.

27. Guo H, Mendrikahy JN, Xie L, Deng J, Lu Z, Wu J, et al. Transcriptome analysis of neo-tetraploid rice reveals specific differential gene expressions associated with fertility and heterosis. Sci Rep. 2017;7:40139.

28. Jin JJ, Sun YW, Qu J, Syah R, Lim CH, Alfiko Y, et al. Transcriptome and functional analysis reveals hybrid vigor for oil biosynthesis in oil palm. Sci Rep. 2017;7:439

29. Cornish K. Similarities and differences in rubber biochemistry among plant species. Phytochemistry. 2001;57:1123-34.

30. Paardekooper E. Exploitation of the rubber tree. Rubber. 1989;5:349-414

31. Varghese YA, John A, Premakumari D, Panikkar AON, Sethuraj MR. Early evaluation in Hevea: growth and yield at the juvenile phase. Ind J Nat Rubber Res. 1992;6:19-23.

32. Mao CL, Yang $Y$, Wu $Y$, Ni SB. Variation analysis on growth traits in seedlings from stock families of Hevea brasiliensis. Trop Agri Sci Technol. 2012;35:5-8.

33. Zhou LJ, Wang J, Chen XH, Lin WF. A comparative study on the different budling of Hevea brasiliensis in the immature period. Chinese Agricultural Sci Bullet2013;29:26-29.

34. Sankariammal L, Mydin KK. Heterosis for growth and test tap yield in Wickham $\times$ Amazonian hybrids of Hevea brasiliensis. In: International rubber Research and Development Board (IRRDB) international workshop on tree breeding. Brazil: Michelin Plantations, Bahia; 2011.

35. Doyle J. Isolation of plant DNA from fresh tissue. Focus. 1990;12:13-5.

36. Grabherr MG, Haas BJ, Yassour M, Levin JZ, Thompson DA, Amit I, et al. Fulllength transcriptome assembly from RNA-Seq data without a reference genome. Nat Biotechnol. 2011;29:644-52.

37. Li B, Dewey CN. RSEM: accurate transcript quantification from RNA-Seq data with or without a reference genome. BMC Bioinform. 2011;12:323.

38. Anders $S$, Huber W. Differential expression analysis for sequence count data. Genome Biol. 2010;11:R106

39. Rapp RA, Udall JA, Wendel JF. Genomic expression dominance in allopolyploids. BMC Biol. 2009;7:18.

40. Wang Z, Gerstein M, Snyder M. RNA-Seq: a revolutionary tool for transcriptomics. Nat Rev Genet. 2009:10:57-63.

41. Ozsolak F, Milos PM. RNA sequencing: advances, challenges and opportunities. Nat Rev Genet. 2011;12:87-98.

42. Van Verk MC, Hickman R, Pieterse CMJ, Van Wees SCM. RNA-Seq: revelation of the messengers. Trends Plant Sci. 2013;18:175-9.

43. Chow KS, Mat-Isa MN, Bahari A, Ghazali AK, Alias H, Zainuddin ZM, Hoh CC, Wan KL. Metabolic routes affecting rubber biosynthesis in Hevea brasiliensis latex. J Exp Bot. 2012;63:1863-71.

44. Gébelin V, Leclercq J, Kuswanhadi Argout X, Chaidamsari T, Hu SN, et al. The small RNA profile in latex from Hevea brasiliensis trees is affected by tapping panel dryness. Tree Physiol. 2013;33:1084-98.

45. Chao JQ, Chen YY, Wu SH, Tian WM. Comparative transcriptome analysis of latex from rubber tree clone CATAS 8-79 and PR 107 reveals new cues for the regulation of latex regeneration and duration of latex flow. BMC Plant Biol. 2015;5:104.

46. Li DJ, Hao LL, Liu H, Zhao MM, Deng Z, Li Y, et al. Next-generation sequencing, assembly and comparative analyses of the latex transcriptomes from two elite Hevea brasiliensis varieties. Tree Genet Genomes. 2015;11:98.

47. Wei F, Luo SQ, Zheng QK, Qiu J, Yang WF, Wu M, et al. Transcriptome sequencing and comparative analysis reveal long-term flowing mechanisms in Hevea brasiliensis latex. Gene. 2015;556:153-62.

48. Li HL, Guo D, Zhu JH, Wang Y, Chen XT, Peng SQ. Comparative transcriptome analysis of latex reveals molecular mechanisms underlying increased rubber yield in Hevea brasiliensis self-rooting juvenile clones. Front Plant Sci. 2016:7:1204

49. Liu JP, Zhuang YF, Guo XL, Li YJ. Molecular mechanism of ethylene stimulation of latex yield in rubber tree (Hevea brasiliensis) revealed by de novo sequencing and transcriptome analysis. BMC Genomics. 2016;17:257.

50. Makita Y, Ng KK, Veera Singham G, Kawashima M, Hirakawa H, Sato S, et al. Large-scale collection of full-length cDNA and transcriptome analysis in Hevea brasiliensis. DNA Res. 2017;24:159-67.

51. Li DJ, Deng Z, Qin B, Liu XH, Men ZH. De novo assembly and characterization of bark transcriptome using Illumina sequencing and development of EST-SSR markers in rubber tree (Hevea brasiliensis Muell. Arg.). BMC Genomics. 2012;13:192.

52. Li DJ, Wang XC, Deng Z, Liu H, Yang H, He GM. Transcriptome analyses reveal molecular mechanism underlying tapping panel dryness of rubber tree (Hevea brasiliensis). Sci Rep. 2016:6:23540.

53. Mantello CC, Cardoso-Silva CB, da Silva CC, de Souza LM, Scaloppi Junior EJ, de Souza GP, et al. De novo assembly and transcriptome analysis of the rubber tree (Hevea brasiliensis) and SNP markers development for rubber biosynthesis pathways. PLoS One. 2014;9:e102665.

54. Liu JP, Xia ZQ, Tian XY, Li YJ. Transcriptome sequencing and analysis of rubber tree (Hevea brasiliensis Muell.) to discover putative genes associated with tapping panel dryness (TPD). BMC Genomics. 2015;16:398.

55. Fang YJ, Mei HL, Zhou BH, Xiao XH, Yang M, Huang YC, Long XY, Hu SN, Tang CR. De novo transcriptome analysis reveals distinct defense mechanisms by young and mature leaves of Hevea brasiliensis (Para rubber tree). Sci Rep. 2016;6:33151.

56. Hedgecock D, Lin JZ, DeCola S, Haudenschild CD, Meyer E, Manahan DT, Bowen B. Transcriptomic analysis of growth heterosis in larval Pacific oysters (Crassostrea gigas). Proc Natl Acad Sci U S A. 2007;104:2313-8.

57. Song GS, Zhai HL, Peng YG, Zhang L, Wei G, Chen XY, et al. Comparative transcriptional profiling and preliminary study on heterosis mechanism of super-hybrid rice. Mol Plant. 2010;3:1012-25

58. Stupar RM, Springer NM. Cis-transcriptional variation in maize inbred lines B73 and Mo17 leads to additive expression patterns in the F1 hybrid. Genetics. 2006;173:2199-210.

59. Li XH, Wei YL, Nettleton D, Brummer EC. Comparative gene expression profiles between heterotic and non-heterotic hybrids of tetraploid Medicago sativa. BMC Plant Biol. 2009;9:107.

60. Groszmann M, Gonzalez-Bayon R, Greaves IK, Wang L, Huen AK, Peacock WJ, et al. Intraspecific Arabidopsis hybrids show different patterns of heterosis despite the close relatedness of the parental genomes. Plant Physiol. 2014; 166:265-80.

61. Groszmann M, Gonzalez-Bayon R, Lyons RL, Greaves IK, Kazan K, Peacock WJ, et al. Hormone-regulated defense and stress response networks contribute to heterosis in Arabidopsis F1 hybrids. Proc Natl Acad Sci U S A. 2015;112: 6397-406.

62. Miller M, Song Q, Shi X, Juenger TE, Chen ZJ. Natural variation in timing of stress-responsive gene expression predicts heterosis in intraspecific hybrids of Arabidopsis. Nat Commun. 2015;6:7453.

63. Todesco M, Balasubramanian S, Hu TT, Traw MB, Horton M, Epple $P$, et al. Natural allelic variation underlying a major fitness trade-off in Arabidopsis thaliana. Nature. 2010;465:632-6.

64. Kempel A, Schaedler M, Chrobock T, Fischer M, van Kleunen M. Tradeoffs associated with constitutive and induced plant resistance against herbivory. Proc Natl Acad Sci U S A. 2011;108:5685-9.

65. Huot B, Yao J, Montgomery BL, He SY. Growth-defense tradeoffs in plants: a balancing act to optimize fitness. Mol Plant. 2014;7:1267-87.

66. Wang L, Greaves IK, Groszmann M, Wu LM, Dennis ES, Peacock WJ. Hybrid mimics and hybrid vigor in Arabidopsis. Proc Natl Acad Sci U S A. 2015;112: 4959-67.

67. Lisec J, Meyer RC, Steinfath M, Redestig H, Becher M, Witucka-Wall H, et al. Identification of metabolic and biomass QTL in Arabidopsis thaliana in a parallel analysis of RIL and IL populations. Plant J. 2008;53:960-72.

68. Lisec J, Steinfath M, Meyer RC, Selbig J, Melchinger AE, Willmitzer L, et al. Identification of heterotic metabolite QTL in Arabidopsis thaliana RIL and IL populations. Plant J. 2009:59:777-88.

69. Ge XM, Chen WH, Song SH, Wang WW, Hu SN, Yu J. Transcriptomic profiling of mature embryo from an elite super-hybrid rice LYP9 and its parental lines. BMC Plant Biol. 2008;8:114.

70. Zhai $R$, Feng $Y$, Wang $H$, Zhan $X$, Shen $X$, Wu W, et al. Transcriptome analysis of rice root heterosis by RNA-Seq. BMC Genomics. 2013;14:19. 